\title{
Blacklegged Tick or Deer Tick, Ixodes scapularis Say (Arachnida: Acari: Ixodidae) ${ }^{1}$
}

Michael R. Patnaude and Thomas N. Mather ${ }^{2}$

\section{Introduction}

Lyme disease was first recognized in 1975 as a distinct clinical disorder (Steere et al. 1977) and is currently the most frequently reported vector-borne disease in the United States (CDC 1995).

Transmission of the spirochete B. burgdorferi, the causative agent of Lyme disease (Burgdorfer et al. 1982), occurs by the bite of Ixodes ticks. In the United States, the blacklegged tick, Ixodes scapularis Say affects the greatest number of people for three principal reasons: their geographic distribution coincides in the northeastern United States with the greatest concentration of humans (Miller et al. 1990); spirochete infection rates are high, often exceeding 25\% (Burgdorfer et al. 1982, Anderson et al. 1983, Magnarelli et al. 1986); and the geographical range of the tick is spreading (Lastavica et al. 1989, Anderson et al. 1990, Godsey et al. 1987, Davis et al. 1984).

In 1993 it was shown that Ixodes dammini is the same species as I. scapularis and as a result has retained the older name (Oliver et al. 1993). Those who read papers with references to Ixodes dammini should make note of this change.

\section{Distribution}

Ixodes scapularis is found along the east coast of the United States. Florida westward into central Texas forms the lower boundary, although there are reports from Mexico. The upper boundary is located in Maine westward to Minnesota and Iowa. The distribution of I. scapularis is linked to the distribution and abundance of its primary reproductive host, white-tailed deer (O. virginianus) (Wilson et al. 1985, 1988). Only deer or some other large mammal appears capable of supporting high populations of ticks (Duffy et al. 1994). In the northeastern United States, much of the landscape has been altered. Forests were cleared for farming, but were abandoned in the late 1800s and 1900s causing succession of the fields to second-growth forests. These second-growth forests created "edge" habitats which provided appropriate habitat for deer resulting in increased populations (Severinghaus and Brown 1956) and thus, may have increased populations of the blacklegged tick.

1. This document is EENY-143, one of a series of Featured Creatures from the Entomology and Nematology Department, Florida Cooperative Extension Service, Institute of Food and Agricultural Sciences, University of Florida. Published: July 2000. Revised: June 2003. This document is also available on Featured Creatures Website at http://creatures.ifas.ufl.edu. Please visit the EDIS Website at http://edis.ifas.ufl.edu. Additional information on these organisms, including many color photographs, is available at the Entomology and Nematology Department website at http://entnemdept.ifas.ufl.edu/.

2. Michael R. Patnaude, Entomology and Nematology Department, University of Florida, Institute of Food and Agricultural Sciences, Gainesville, FL and Thomas N. Mather, University of Rhode Island, Kingston, RI 02881.

The Institute of Food and Agricultural Sciences is an equal opportunity/affirmative action employer authorized to provide research, educational information and other services only to individuals and institutions that function without regard to race, color, sex, age, handicap, or national origin. For information on obtaining other extension publications, contact your county Cooperative Extension Service office. Florida Cooperative Extension Service/Institute of Food and Agricultural Sciences/University of Florida/Christine Taylor Waddill, Dean. 


\section{Description}

Adult deer ticks have no white markings on the dorsal area nor do they have eyes or festoons. They are about $3 \mathrm{~mm}$ and dark brown to black in color. Adults exhibit sexual dimorphism. Females typically have the area behind the scutum with an orange to red color.

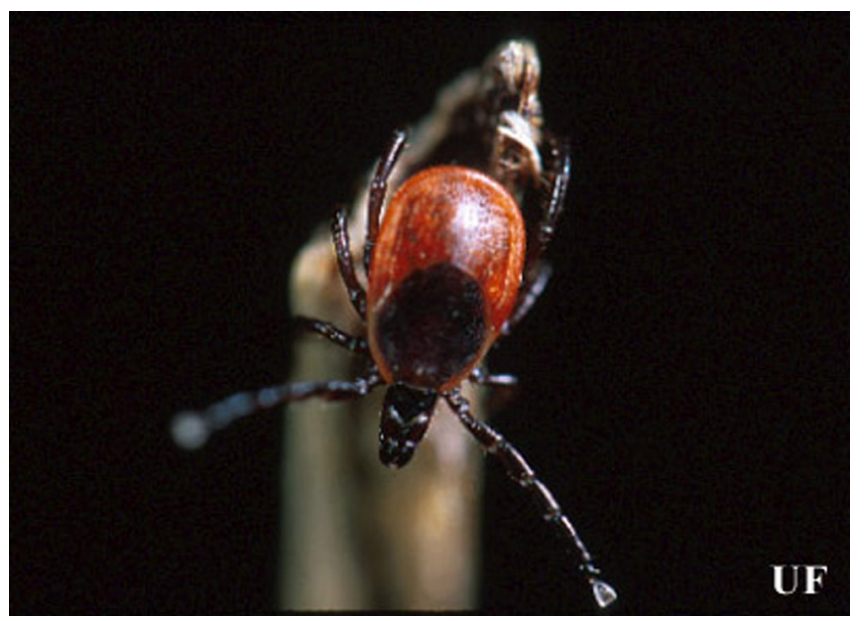

Figure 1. Female blacklegged tick, Ixodes scapularis Say, questing on a stick. Credits: Michael Patnaude, University of Florida

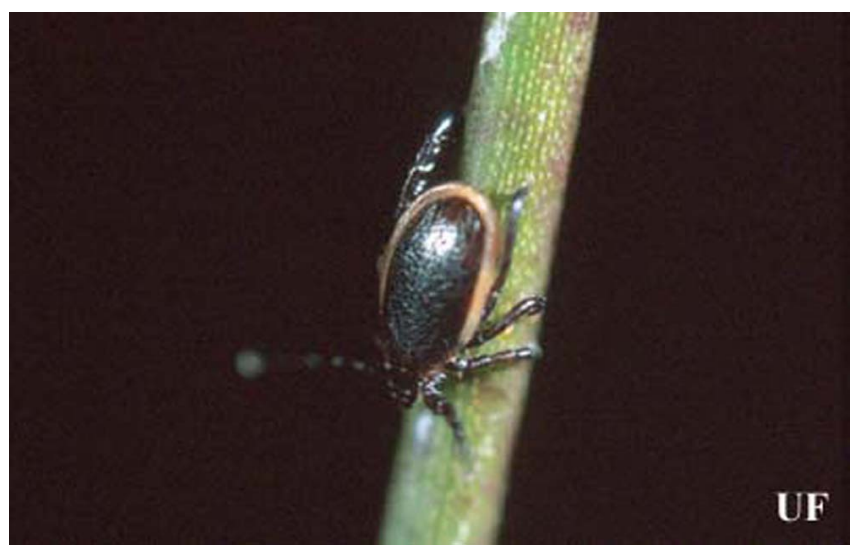

Figure 2. Male blacklegged tick, Ixodes scapularis Say. Credits: Michael Patnaude, University of Florida

\section{Life Cycle}

I. scapularis is a three-host tick; each mobile stage feeds upon a different host animal. In June and July, eggs deposited earlier in the spring hatch into tiny six-legged larvae. Peak larval activity occurs in August, when larvae attach and feed on a wide variety of mammals and birds, primarily on white-footed mice (Peromyscus leucopus) (Anderson and Magnarelli 1980). After feeding for three to five days,

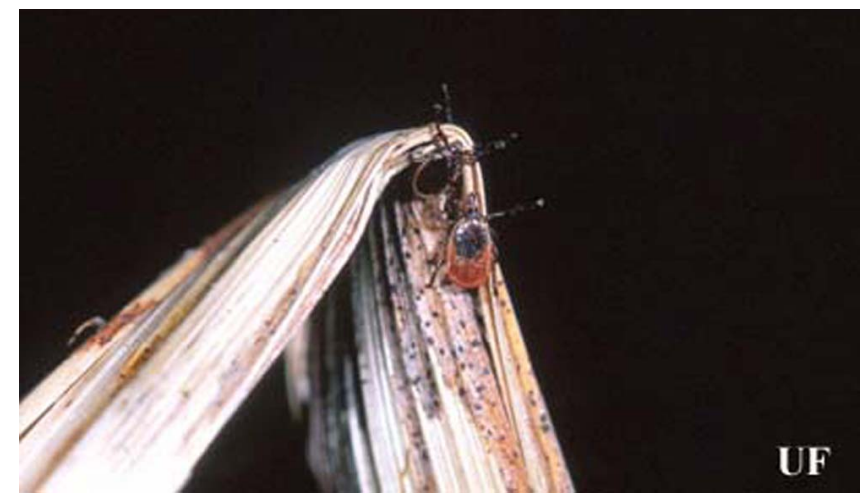

Figure 3. Two male and one female blacklegged tick, Ixodes scapularis Say, questing on vegetation. Only the legs are visible from the second male tick, which is on the opposite side of the vegetation. Credits: Michael Patnaude, University of Florida

engorged larvae drop from the host to the ground where they overwinter. In May, larvae molt into nymphs, which feed on a variety of hosts for three to four days. In a similar manner, engorged nymphs detach and drop to the forest floor where they molt into the adult stage, which becomes active in October. Adult ticks remain active through the winter on days when the ground and ambient temperatures are above freezing. Adult female ticks feed for five to seven days while the male tick feeds only sparingly, if at all. Adult ticks feed on large mammals, primarily upon white-tailed deer (Odocoileus virginianus) (Piesman et al. 1979, Carey et al. 1980, Wilson et al. 1990). Beginning in May, engorged adult females typically lay between 1000-3000 eggs on the forest floor at the site where they detached from their hosts.

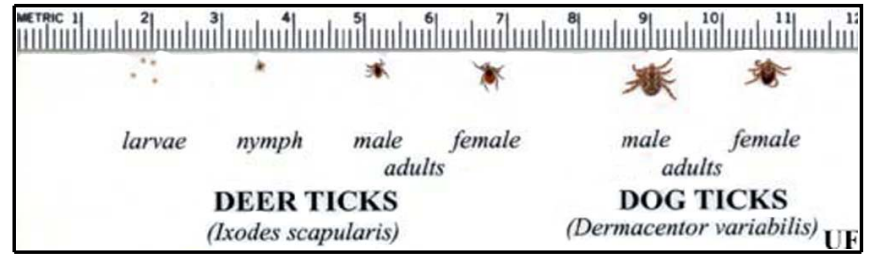

Figure 4. The life cycle and approximate sizes of the blacklegged tick, Ixodes scapularis Say, compared with the American dog tick, Dermacentor variabilis Say. Credits: Michael Patnaude, University of Florida

Mortality rates for ticks are high. Tick death is caused by density-dependent factors such as parasites, pathogens, and predators, all of which appear to have little impact on tick populations (Roberts et al. 1983, Matthewson 1984, Mather et al. 1987a). Density-independent factors causing tick mortality include a variety of adverse climatic and 
microclimate conditions, which can influence temperature and humidity and have the greatest impact on tick survival (Bertrand and Wilson 1996). Due to their low probability of finding a host, starvation also would be a major mortality factor of ticks. Host immunity and grooming activity also may affect mortality (Randolph 1979, Brown 1988).

\section{Medical Significance}

The blacklegged tick, Ixodes scapularis, is an important vector of the Lyme disease spirochete, Borrelia burgdorferi, as well as the agents of human babesiosis, Babesia microti, and human granulocytic ehrlichiosis (HGE) (Des Vignes and Fish 1997). A significant feature in the transmission dynamics of $B$. burgdorferi is the importance of the nymphal stage's activity preceding that of the larvae which allows for an efficient transmission cycle (Spielman et al. 1985, Wilson and Spielman 1985). Before and during larval tick feeding, the naturally infected nymphs transmit

B. burgdorferi to reservoir hosts. The newly hatched spirochete-free larvae (Piesman et al. 1986a) acquire the bacteria from the reservoir host and retain infection through the molting process. In the springtime, nymphs derived from infected larvae transmit infection to susceptible animals, which will serve as hosts for larvae later in the summer (Fish 1993).

Human exposure to blacklegged ticks is greatest during the summer months when high nymphal $I$. scapularis activity and human outdoor activity coincide. Their small size, their vastly greater abundance over the adult stages and the difficulty in recognizing their bites (Berger 1989) tends to make nymphs the most important stage to consider for reducing disease risk.

\section{Surveillance}

Researchers currently use seven techniques to sample for ticks:

1. dragging cloth flags over vegetation,

2. carbon dioxide-baited traps,

3. collecting from host animals,
4. collecting from the investigator's clothing

(Ginsberg and Ewing 1989a),

5. using live, caged sentinel hosts,

6. aspiration of nests and most recent by

7. using artificial nest-box traps (Wilson 1994).

Flagging for ticks involves using a cotton flannel or other fabric attached to a wooden pole. The cloth is either hung at one end in a flag configuration and dragged, or is attached to the middle and dragged by rope tied to each end of the wooden pole (Ginsberg and Ewing 1989a). The use of flags is the preferred method when collecting larval and nymphal Ixodes ticks as it samples host- seeking ticks in the leaf litter over a quantifiable distance or exposure. Traps baited with carbon dioxide are typically made out of a covered plastic tub mounted to a wooden base. Evenly spaced holes are made on the sides of the tub at the bottom, and dry ice is placed into the container daily during the trap's operation (Ginsberg and Ewing 1989a). As a surveillance tool, CO2 traps are generally unsuccessful for collecting Ixodes scapularis as these ticks exhibit limited horizontal movement (Falco and Fish 1991).

To sample host-attached ticks, hosts typically are captured in live-capture traps after which animals are anesthetized and attached ticks are counted (Ginsberg and Ewing 1989b). A variation of this method involves holding the captured animals in wire mesh cages over pans of water until ticks detach (Mather and Mather 1990). Collecting ticks from the investigator while walking involves wearing cotton pants tucked into socks, and the collector removes attached ticks periodically while walking through the sampling area (Ginsberg and Ewing 1989a). This latter method is particularly effective for sampling adult ticks. Caged hosts can be used to attract questing ticks in a manner similar to $\mathrm{CO} 2$ trapping with the same effectiveness. Ticks can also be collected by aspirating excavated burrows and nests of hosts (Logan et al. 1993). This aspiration method is best used for collecting ticks associated with birds. Ticks can be retrieved from artificial nest-box traps when hosts inhabit the nest-box and ticks and other ectoparasites drop onto a sticky substrate which can be removed at a later time (Wilson 1994). 


\section{Management}

Management of ticks and Lyme disease usually involve several strategies including surveillance, personal protection and vector reduction.

Surveillance is needed to identify areas for control and to prioritize management efforts. Ticks are surveyed by capturing mammals and birds and by sampling vegetation as described above. Pathogen infection rates of ticks or animals can be determined using various methods such as a direct fluorescent antibody procedure (Burgdorfer et al. 1982, Piesman et al. 1986b) and an indirect fluorescent antibody technique (Burgess et al. 1986, Godsey et al. 1987). Pathogen distribution's can be assessed using serological surveys (Daniels et al. 1993). Disease also can be tracked by monitoring cases from hospitals and other health agencies (White 1993).

In any event the public is always informed through the media with the most recent information so that they can implement personal protection methods. These methods include avoidance of tick habitat and taking precautions when entering these high risk areas. Socks pulled over the cuffs of the trousers, wearing light-colored clothing, walking in the center of trails, frequently checking clothes, application of repellents such as DEET containing products and examination of body at the end of the day are the precautions that one should take in tick habitat (Stafford 1989).

There are three basic methods for vector management: reduce immigration, increase mortality, and reduce reproduction. Restricting the movement of infested hosts into an area reduces the immigration of ticks since they cannot move far on their own. Birds are difficult to restrict but by reducing food supplies and preferred vegetation their migration through an area can be decreased. The density and movement of rodents, which do not travel large distances, may be influenced by altering habitat to reduce brush, stacked wood and food sources. Large mammals such as deer can be discouraged by using plants that do not attract them, fencing (Olkowski et al. 1990) and deer repellents (Daniels et al. 1989, Wilson et al 1990). Increasing the mortality of the tick is an effective strategy. Targeting areas of high tick densities with pesticides is effective if it can reach the ticks
(Schulze et al. 1987, Stafford 1991). Unfortunately, leaf litter provides a barrier to application. Pheromones combined with acaricides seem to have potential by targeting the tick with little effect to other arthropods, but this method has not been tested on I. scapularis (Wilson and Deblinger 1993).

The destruction of vegetation by burning is another procedure that can reduce tick numbers (Wilson 1986), but this may not kill the greatest population of spirochete-infected ticks which are protected in the dens of rodents underground (Mather et al. 1993). Altering the habitat around ones own lawn can have a positive effect (Wilson 1986). Mowing, raking leaves, trimming back shrubs, and/or using pesticide treated wood chips can reduce favorable habitat for ticks by exposing these areas to lower humidity or pesticides (Mather in press). Eliminating the hosts of ticks reduces tick populations as well. Deer populations may be easier to reduce than those of rodents or birds. Experiments where deer were eliminated resulted in a decrease in the populations of larvae and nymphs (Wilson et al. 1988). Efforts have been made to target the immature ticks on the host with success using nesting material laced with pesticides (Mather et al. 1987b, 1988) or boxes containing pesticides with a food lure (Sonenshine and Haines 1985). The feeding success of ticks can also be reduced by host immunity (Brown 1988). Tick repellents and acaricides used to repel or kill ticks as a control procedure pose difficulty because it requires handling large animal hosts (deer). Bait stations, where deer are lured to food to inoculate themselves with an acaricide or ingested treatment such as ivermectin show promise. Biological control using predators, parasites or pathogens have not been demonstrated under natural conditions to reduce populations significantly (Mather et al. 1987a, Wilson and Deblinger 1993).

For more information see:

Insect Management Guide for Ticks

Lyme Disease 